\title{
IMITATION MOTIVES IN RETAILERS’ ADOPTION OF THE INTERNET CHANNEL
}

\author{
Ralitza Nikolaeva, ISCTE-Instituto Universitário de Lisboa, Portugal
}

\begin{abstract}
Imitative behavior in technology adoption has been documented in various industries. However, not much attention has been paid to the motivations behind it. Knowledge of the context of an imitation strategy can bring further insights of firms' interpretation of the market situation and the mechanisms of technology diffusion. The study empirically investigates the nature of imitation in the case of retailers' adoption of the Internet as a transaction channel while controlling for economic and firm factors. We combine Lieberman and Asaba's (2006) and Ordanini et al.'s (2008) categorizations to formulate hypotheses aimed at distinguishing between different types of imitation behavior. We use a discrete hazard rate model to analyze the empirical data. In this framework we discuss the importance of accounting for non-linearity by employing a flexible multivariable model-building technique based on fractional polynomials. The predominant evidence points to information-based imitation with search for effectiveness motives. We discuss the strategic implications of the observed mimetic behavior and its links to market signal framing.
\end{abstract}

References available upon request 